Tropical Journal of Pharmaceutical Research September 2021; 20 (9): 1875-1880

ISSN: $1596-5996$ (print); 1596-9827 (electronic)

(C) Pharmacotherapy Group, Faculty of Pharmacy, University of Benin, Benin City, 300001 Nigeria.

Original Research Article

http://dx.doi.org/10.4314/tjpr.v20i9.13

\title{
Trichostatin A reduces nerve cell apoptosis in depressive rats by regulating CREB/BDNF signaling pathway
}

\author{
Lianyu Jin*, Ying Bao \\ Mental Health Department, The Second Hospital of Yinzhou District, Ningbo 315000, China
}

*For correspondence: Email: 1611085047@qq.com; Tel: +86-015168582553

Sent for review: 24 June 2021

Revised accepted: 23 August 2021

\begin{abstract}
Purpose: To investigate the influence of trichostatin A on nerve cell apoptosis in depressive rats and to explore the probable molecular mechanism of action.

Methods: A total of 36 Sprague-Dawley rats weighing $200-220 \mathrm{~g}$ were divided into sham group ( $n=$ 12), model group $(n=12)$ and trichostatin group $(n=12)$ by randomization. The protein expressions of phosphorylated CAMP responsive element-binding protein ( $p-C R E B)$ and BDNF, as well as the mRNA expression levels of B-cell lymphoma-2 (Bcl-2) and caspase-3 in each group of rat hippocampus were determined by Western blotting and quantitative reverse transcription-polymerase chain reaction ( $q R T$ $P C R)$, respectively. The apoptosis of nerve cells in the brain tissues of the rats was labeled using terminal deoxynucleotidyl transferase-mediated dUTP nick end labeling (TUNEL) staining.

Results: Compared with those in the sham group, the degree of sucrose preference decreased markedly, while the immobility time after forced swimming test was extended, and the relative expression levels of $p-C R E B$ and BDNF proteins in the hippocampus declined $(p<0.05)$. The mRNA levels of Bcl-2 and caspase-3 and cell apoptosis rate were increased in the model group $(p<0.05)$. In comparison with the model group, the trichostatin group exhibited increased sucrose preference degree, shortened immobility time following a forced swimming test, and elevated relative expression levels $p$ CREB and BDNF proteins in the hippocampus $(p<0.05)$, but lowered mRNA levels of Bcl-2 and caspase-3 and cell apoptosis rate, displaying statistically significant differences $(p<0.05)$.

Conclusion: Trichostatin A reduces cell apoptosis and ameliorates the depression-like behaviors of rats via the regulation of $C R E B / B D N F$ signaling pathway. These findings provide new insights into Trichostatin A for the management of depression.
\end{abstract}

Keywords: Trichostatin A, Depressive, CREB/BDNF signaling pathway, Nerve cell apoptosis

\begin{abstract}
This is an Open Access article that uses a funding model which does not charge readers or their institutions for access and distributed under the terms of the Creative Commons Attribution License (http://creativecommons.org/licenses/by/4.0) and the Budapest Open Access Initiative (http://www.budapestopenaccessinitiative.org/read), which permit unrestricted use, distribution, and
\end{abstract} reproduction in any medium, provided the original work is properly credited.

Tropical Journal of Pharmaceutical Research is indexed by Science Citation Index (SciSearch), Scopus, International Pharmaceutical Abstract, Chemical Abstracts, Embase, Index Copernicus, EBSCO, African Index Medicus, JournalSeek, Journal Citation Reports/Science Edition, Directory of Open Access Journals (DOAJ), African Journal Online, Bioline International, Open-J-Gate and Pharmacy Abstracts

\section{INTRODUCTION}

Depression, one of the leading causes of disability in the world, is characterized by selfdefiance, divergent thinking, low spirits [1]. According to statistics, about one-sixth of people die of depression, aggravating the social medical burden around the globe and posing a greater threat to the quality of life of patients with cardiovascular disease [2]. The pathological mechanism of depression involves the abnormality of monoaminergic and glutamatergic systems, increase in inflammatory responses, aberration of the HPA axis and decrease in 
neurogenesis and neural plasticity [3]. Histone deacetylases (HDACs) are capable of regulating chromatin function by means of histone acetylation and play crucial roles in learning, memory and synaptic plasticity [4]. In the culture of cortical neurons of mice, inhibiting HDAC6 via genetic and pharmacologic approaches can maintain the survival and regeneration of neurons under the condition of oxidative stress. However, in the rat model of chronic depression induced by social pressure, the expression of HDAC5 is raised in the prefrontal cortex [5], while trichostatin $A$, as a HDAC inhibitor, can improve the depressive behavior $[6,7]$.

Brain-derived neurotrophic factor (BDNF) has been more and more frequently studied and applied in animal models of depression, which is often distributed in the hippocampus and involved in the hippocampal neurogenesis and development. It has been revealed that the cAMP responsive element-binding protein (CREB)/BDNF signaling pathway is downregulated in the brain hippocampus of depression model [8]. In addition, a large number of studies have illustrated that CREB has certain associations with the pathogenesis of depression and the action mechanism of antidepressant drugs.

After treatment with trichostatin $A$, the expressions of BDNF and phosphorylated CREB (p-CREB) in hippocampal tissues are increased notably compared with those in depressive rats [9], suggesting that the antidepressant-like effect of trichostatin A may be closely related to the CREB/BDNF signaling pathway. Moreover, the CREB/BDNF pathway represents an important signal transduction pathway in the regulation of inflammation and cell apoptosis. Therefore, this research aims to investigate whether trichostatin $A$ affects the nerve cell apoptosis, and improves the depressive symptoms through the CREB/BDNF pathway in depressive rats, thereby providing clarity on the mechanism action of trichostatin A in depressive rats.

\section{EXPERIMENTAL}

\section{Animal experiments and grouping}

A total of 36 Specific Pathogen-Free (SPF) male Sprague-Dawley rats weighing 200 - $220 \mathrm{~g}$ were raised in an animal room at $20-25{ }^{\circ} \mathrm{C}$, with relative humidity of $55-65 \%$ and $12 / 12 \mathrm{~h}$ light/dark cycles. The laboratory rats were fed adaptively for 1 week before operation, and they had free access to water and feed. Moreover, they were deprived of feed instead of water for $12 \mathrm{~h}$ before operation, and then the operation was performed. The 36 rats were divided into sham group $(n=12)$, model group $(n=12)$ and trichostatin group $(n=12)$ using a random number table. This study was approved by the Animal Ethics Committee of The Second Hospital of Yinzhou District Animal Center, and followed international guidelines for animal studies.

\section{Establishment of model}

Chronic unpredictable mild stress (CUMS) was used for modeling for $21 \mathrm{~d}^{10}$, and the rats were fed in separate cages (1 rat/cage). As for modeling, the rats were stimulated by randomly selected unpredictable stress factors, including foot shock for $5 \mathrm{~min}$, tail clamping for $5 \mathrm{~min}$, swimming in hot water at $45^{\circ} \mathrm{C}$ for 5 min, fasting + day-night reversal, high-speed horizontal vibrating for $5 \mathrm{~min}$, water deprivation and swimming in cold water at $4^{\circ} \mathrm{C}$ for 5 min (once a day). Each stimulation was employed discontinuously and once every 7 days on average. In trichostatin group, trichostatin A (25 $\mathrm{mg} / \mathrm{kg}$ ) was intraperitoneally injected for 21 consecutive days. The rats in sham group were fed with normal diet, with 5 rats in each cage.

\section{Main reagents and devices}

Trichostatin A (Sigma-Aldrich, St. Louis, MO, USA), p-CREB, BDNF, B-cell lymphoma-2 (Bcl2) and Caspase-3 primary antibodies (Abcam, Cambridge, MA, USA), terminal deoxynucleotidyl transferase-mediated dUTP nick end labeling (TUNEL) assay kit (Beyotime Biotechnology, Shanghai, China), quantitative polymerase chain reaction (qPCR) kit (Vazyme Biotech Co., Ltd., Nanjing, China), immunohistochemistry kit (Fuzhou Maxin Biotechnologies, Fuzhou, China), bicinchoninic acid (BCA) protein assay kit (Beyotime Biotechnology, Shanghai, China), fluorescence qPCR instrument (ABI 7500, Applied Biosystems, Foster City, CA, USA), ImageLab image analysis system, Leica DM4000B LED microscope (Leica, Wetzlar, Germany), and YP601N type electronic balance (Sincere Dedication of Science And Technology Innovation, Shanghai, China).

\section{Sucrose preference test}

At 21 days after stress stimulation, the rats were placed in a sound-proof and quiet room for adaptation for 2 days. On the $1^{\text {st }}$ day, 2 bottles of equal amount of pure water were put in each cage, and 2 bottles of equal amount of $1 \%$ sucrose water were provided on the $2^{\text {nd }}$ days. The rats could eat food freely during the adaptive feeding. The sucrose preference test was conducted from the $3^{\text {rd }}$ day, where one bottle of 
pure water and one bottle of $1 \%$ sucrose water in equal weights were provided. $2 \mathrm{~h}$ later, the test was stopped, and the weight of the 2 bottles was measured, to calculate sucrose preference degree $(P)$ as in Eq 1.

$P(\%)=(S / L) 100 \ldots \ldots \ldots(E q 1)$

where $S$ is sucrose consumption and $L$ is total liquid consumption.

\section{Forced swimming test}

The rats adapted to the environment at $24 \mathrm{~h}$ before experiment. Each rat was placed in a separate clear glass container (diameter: $20 \mathrm{~cm}$, water level: $40 \mathrm{~cm}$, water temperature: 23 $25^{\circ} \mathrm{C}$ ) for $15 \mathrm{~min}$ of adaptation. After $24 \mathrm{~min}$, the forced swimming test was implemented. Each rat swam adaptively for $2 \mathrm{~min}$, and then the immobility time of the rat within the following 4 min was recorded, during which the rat floated in the water, with the head out of water for breathing. After the test, the rat was wiped dry using a towel and placed back in the cage.

Determination of messenger ribonucleic acid (mRNA) levels of Bcl-2 and Caspase-3 via qPCR

The RNA was extracted from the hippocampus of the rats in each group, which concentration was determined by a spectrophotometer. Later, the RNA was reversely transcribed into complementary deoxyribonucleic acid (cDNA) using the transcription kit. The primer sequences were shown in Table 1. Amplification was conducted using a $20 \mu \mathrm{L}$ reaction system in an amplification instrument. Reverse transcription conditions: denaturation at $94^{\circ} \mathrm{C}$ for $45 \mathrm{~s}$ and annealing at $55{ }^{\circ} \mathrm{C}$ for $45 \mathrm{sec}$ for 30 cycles, followed by extension at $72{ }^{\circ} \mathrm{C}$ for $45 \mathrm{sec}$. After that, cDNA was stored in a refrigerator at $-20{ }^{\circ} \mathrm{C}$. The relative expression of target genes was calculated by $2^{-\Delta \Delta C t}$ method with $\mathrm{GAPDH}$ as the reference gene.

Measurement of protein expressions of $p$ CREB and BDNF in hippocampal tissues

The rats were put on ice after sacrifice via decapitation to quickly separate the hippocampus, which was subjected to ultrasonic homogenization and centrifugation twice to obtain the supernatant. Then the protein concentration was quantified using BCA method, and the protein was separated via gel electrophoresis and transferred onto a membrane, followed by sealing at room temperature and incubation with p-CREB and BDNF antibodies at $4{ }^{\circ} \mathrm{C}$ overnight. The next day, the membrane was washed, incubated with horseradish peroxidase-labeled secondary antibodies and washed again. Finally, the image was developed and exposed using electrochemiluminescence (ECL), and the ratio of grayscale value of target protein bands to that of GADPH protein bands was analyzed using ImageJ software.

\section{Evaluation of cell apoptosis}

TUNNEL staining was employed. The brain slices were deparaffinized in xylene and then dehydrated in graded alcohol. After antigen retrieval with citric acid, the membrane was ruptured, stained using the TUNNEL staining kit, mounted in anti-fade mounting medium and observed under a fluorescence microscope. Finally, the positive cells were counted, and the apoptosis rate $(R)$ was calculated.

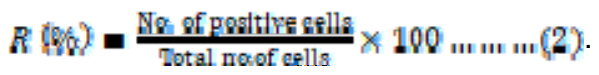

\section{Statistical analysis}

SPSS 19.0 software (IBM, Armonk, NY, USA) was used for data recording and processing. Differences between two groups were analyzed by using the Student's t-test. Comparison between multiple groups was done using Oneway ANOVA test followed by Post Hoc Test (Least Significant Difference). Those not meeting normal distribution were compared to nonparametric test, and $p<0.05$ suggested that the difference was statistically significant.

\section{RESULTS}

\section{Degree of sucrose preference}

The sucrose preference degree was lowered remarkably in the model group compared with that in the sham group,

Table 1: Primers used for $\mathrm{qPCR}$

\begin{tabular}{lll}
\hline Gene name & Forward (5'>3') & Reverse (5'>3') \\
\hline Bcl-2 & 5'-CCCAGCATGCGGCCTCTGTTT-3' & 5'-GGGGGGCA-GGAGGGGCUCA-3' \\
Caspase-3 & 5'-GACCCGGTGCCTCAGGATGC-3' & 5'-GTGGCATGAGCTCTTGATAATG-3' \\
GAPDH & 5'-CCCATCACCATCTTCCAGGA-3' & 5'-CATCGCCCCACTTGATTTTG-3' \\
\hline
\end{tabular}


Table 2: Comparison of degree of sucrose preference, immobility time after the forced swimming test and apoptosis rate of nerve cells among groups

\begin{tabular}{lccc}
\hline Group & $\begin{array}{c}\text { Sucrose preference } \\
\text { degree (\%) }\end{array}$ & Immobility time (s) & Apoptosis rate (\%) \\
\hline Sham group & $71.74 \pm 7.85$ & $88.23 \pm 7.35$ & $16.59 \pm 1.81$ \\
Model group & $36.83 \pm 3.57^{*}$ & $158.82 \pm 13.97^{*}$ & $49.78 \pm 5.21^{*}$ \\
Trichostatin group & $61.59 \pm 5.12^{\#}$ & $85.29 \pm 6.62^{\#}$ & $27.02 \pm 3.09^{\#}$ \\
\hline${ }^{*} P<0.05$ vs. sham group, \#p $<0.05$ vs. model group & &
\end{tabular}

while it was raised evidently in the trichostatin group in comparison with that in the model group $(p<0.05)$, and the differences were statistically significant (Table 2).

\section{Forced swimming}

The immobility time after the forced swimming test was extended in the model group in comparison with that in the sham group, while it was shortened in the trichostatin group compared with that in the model group ( $p<$ 0.05), displaying statistically significant differences (Table 2).

\section{mRNA levels of Bcl-2 and caspase-3}

The model group rats exhibited higher mRNA levels of $\mathrm{Bcl}-2$ and Caspase- 3 than the sham group, and the trichostatin group manifested lower mRNA levels of Bcl-2 and Caspase-3 than the model group $(p<0.05)$ (Figure 1$)$.

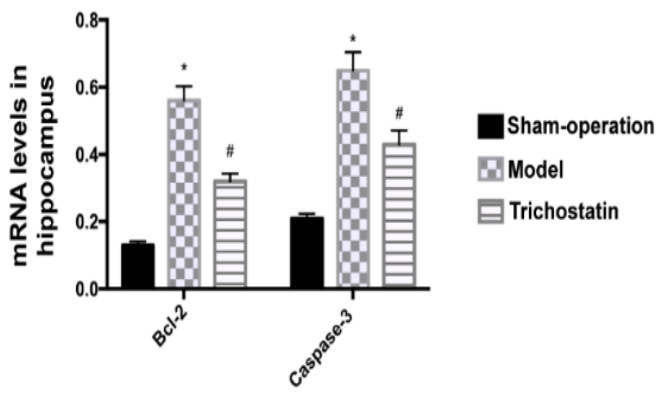

Figure 1: Comparison of mRNA levels in hippocampus among groups. ${ }^{*} P<0.05$ vs. sham group, ${ }^{\#} p<0.05$ vs. model group Relative expression levels of $p$-CREB and
BDNF proteins in hippocampal tissues

Compared with the sham group, the model group had decreased relative protein expression levels of $p$-CREB and BDNF in hippocampus $(p<$ 0.05 ), but in comparison with the model group, the trichostatin group had increased relative expression levels of $p$-CREB and BDNF proteins in hippocampus $(p<0.05)$, (Figure 2 and Figure 3).

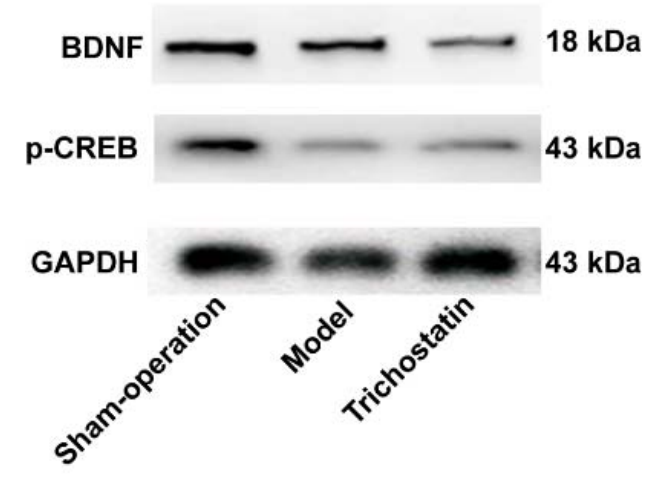

Figure 2: Relative protein expressions in hippocampus

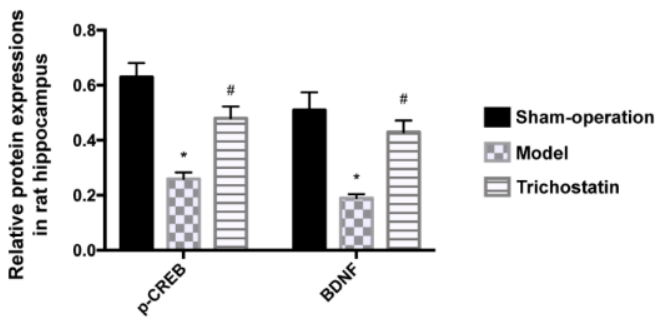

Figure 3: Comparison of relative protein expressions in rat hippocampus among groups. ${ }^{*} P<0.05$ vs. sham group, ${ }^{\#} p<0.05$ vs. model group

\section{Nerve cell apoptosis in hippocampus of rats}

According to TUNEL staining, the apoptosis rate of nerve cells in the hippocampus was elevated in model group compared with that in both sham group $(p<0.05)$ and trichostatin group $(p<$ $0.05)$, showing statistically significant differences (Table 2).

\section{DISCUSSION}

As a common type of psychiatric disorder, depression is manifested as being in low spirits, loss of interest or pleasure in things, diminution of energy, sense of guilt or decrease in selfworth, insomnia or inappetence, poor concentration and anxiety. According to the Global Burden of Disease Study, depression will become the second leading cause of global disability, and a vital reason for suicide and ischemic heart disease by 2020 . It is essential to develop efficient and rapid antidepressant 
therapies, since the etiology of depression has not been completely understood [11]. Studies have demonstrated that environmental factors will result in mental diseases, and the exposure to chronic stress may alter gene expression and induce functional changes of the neural circuit, thus leading to behavioral abnormalities $[12,13]$.

Epigenetic modifications maintained the gene expression in specific brain regions. Epigenetics refers to the functional changes in DNAs, proteins or RNAs triggered by their covalent modification, which possesses dynamic characteristics and changes along with the environmental stimuli. Epigenetic regulation is the basis of many cellular physiological and pathological processes [14,15]. During the control of multiple post-translational modifications of histones that regulate the transcription of specific genes by focusing on their chromatin structures, histone acetylation and deacetylation are the most common forms of neurological and psychiatric disorders [16]. HDACs, a crucial regulatory enzyme, can resist the effect of HAT by reversing lysine acetylation, which restores the positive charge on lysine to stabilize the local chromatin structure. The dysregulation of HDACs will impair the acetylation and deacetylation and lead to the development of depression [17]. As an inhibitor of HDACs, trichostatin A may become a promising tool for the treatment of depression. Inducing acetylation in the hippocampus by trichostatin A can distinctly enhance the preference for sucrose and reduce the immobility time in forced swimming test of the depressive rats [7].

Studies have elaborated that depression causes changes in BDNF-related signaling pathways, and BDNF expression is regulated by various signaling pathways, including CREB, which is one of the most frequently researched transcription factors that participate in the etiology of depressive and anti-depressive reactions. CREB is not only a regulatory factor in the nucleus but also an important regulatory factor of a variety of intra-cellular signaling pathways in the nervous system [18]. The CREB signal transduction in the hippocampus is correlated with psychosis and impairment of cognitive behavior. Besides, BDNF is one of the major downstream target genes of CREB, while CREB is the most prevalent neurotrophin in brain dysfunction. Moreover, BDNF is also considered as one of the target molecules that have been the most widely studied in controlling brain plasticity, survival and differentiation in the peripheral and central nervous system [19].
The expressions of the BDNF and CREB/BDNF signaling pathways, including BDNF, p-CREB, phosphorylated ERK1/2, phosphorylated AKT and TrkB (a receptor of BDNF), decline dramatically in the hippocampus of depression model [20,21]. It has been elucidated in epigenetic investigations that HDAC6 can affect neuronal and synaptic plasticity by regulating CREB. The expression levels of BDNF and $p-$ CREB in rat hippocampus in trichostatin $A$ group increased remarkably, indicating that trichostatin A probably exerts an antidepressant-like effect by modulating the CREB/BDNF signaling pathway $[9,22]$.

In this research, it was discovered in the rat model of CUMS-induced depression that the expression levels of BDNF and p-CREB in the hippocampus declined and mediated the cell apoptosis. However, the CREB/BDNF signaling pathway was regulated, the cell apoptosis was weakened, and the depression-like behaviors were improved after the treatment with trichostatin A.

\section{CONCLUSION}

Trichostatin A ameliorates cell apoptosis and depression-like behaviors of rats by controlling the CREB/BDNF signaling pathway, which may serve as a new target for the treatment of depression. These findings provide new insights into Trichostatin A for the treatment of depression.

\section{DECLARATIONS}

\section{Conflict of interest}

No conflict of interest is associated with this work.

\section{Contribution of authors}

We declare that this work was done by the authors named in this article and all liabilities pertaining to claims relating to the content of this article will be borne by the authors.

\section{Open Access}

This is an Open Access article that uses a funding model which does not charge readers or their institutions for access and distributed under the terms of the Creative Commons Attribution License (http://creativecommons.org/licenses/by/ 4.0) and the Budapest Open Access Initiative (http://www.budapestopenaccessinitiative.org/rea d), which permit unrestricted use, distribution, 
and reproduction in any medium, provided the original work is properly credited.

\section{REFERENCES}

1. Cui R. Editorial: A Systematic Review of Depression. Curr Neuropharmacol 2015; 13(4): 480.

2. Liu J, Xu ZQ, Yi X, Wang YJ, Zhou HD. A study on related factors of hemodynamic depression in carotid artery stenting. Eur Rev Med Pharmacol Sci 2018; 22(16): 5255-63.

3. Dean J, Keshavan M. The neurobiology of depression: An integrated view. Asian J Psychiatr 2017; 27: 101111.

4. Ferland $C L$, Schrader $L A$. Regulation of histone acetylation in the hippocampus of chronically stressed rats: a potential role of sirtuins. Neurosci 2011; 174: 104-114.

5. Munoz-Cobo I, Erburu MM, Zwergel C, Cirilli R, Mai A, Valente S, Puerta E, Tordera RM. Nucleocytoplasmic export of HDAC5 and SIRT2 downregulation: two epigenetic mechanisms by which antidepressants enhance synaptic plasticity markers. Psychopharmacol (Berl) 2018; 235(10): 2831-246.

6. Machado-Vieira $R$, Salvadore $G$, DiazGranados $N$, Ibrahim L, Latov D, Wheeler-Castillo C, Baumann J, Henter ID, Zarate CJ. New therapeutic targets for mood disorders. Scientific World J 2010; 10: 713-726.

7. Lu Y, Zhang J, Zhang L, Dang S, Su Q, Zhang $H$, Lin T, Zhang $X$, Zhang $Y$, Sun H, Zhu Z, Li H. Hippocampal Acetylation may Improve Prenatal-Stress-Induced Depression-Like Behavior of Male Offspring Rats Through Regulating AMPARs Expression. Neurochem Res 2017; 42(12): 3456-3464.

8. Bian $Y$, Yang $L$, Wang $Z$, Wang $Q$, Zeng $L, X U$ G. Repeated Three-Hour Maternal Separation Induces Depression-Like Behavior and Affects the Expression of Hippocampal Plasticity-Related Proteins in C57BL/6N Mice. Neural Plast 2015; 2015: 627837.

9. Gu S, Jing L, Li Y, Huang JH, Wang F. Stress Induced Hormone and Neuromodulator Changes in Menopausal Depressive Rats. Front Psychiatry 2018; 9: 253.

10. Ren L, Hao X, Min S, Deng J, Chen Q, Chen H, Liu D. Anesthetics alleviate learning and memory impairment induced by electroconvulsive shock by regulation of NMDA receptor-mediated metaplasticity in depressive rats. Neurobiol Learn Mem 2018; 155: 65-77.

11. Duman RS. Neurobiology of stress, depression, and rapid acting antidepressants: remodeling synaptic connections. Depress Anxiety 2014; 31(4): 291-296.

12. Lesse A, Rether K, Groger N, Braun K, Bock J. Chronic Postnatal Stress Induces Depressive-like Behavior in Male Mice and Programs second-Hit Stress-Induced
Gene Expression Patterns of OxtR and AvpR1a in Adulthood. Mol Neurobiol 2017; 54(6): 4813-4819.

13. Nasca $C$, Zelli D, Bigio B, Piccinin S, Scaccianoce S, Nistico $R$, McEwen BS. Stress dynamically regulates behavior and glutamatergic gene expression in hippocampus by opening a window of epigenetic plasticity. Proc Natl Acad Sci USA 2015; 112(48): 14960-14965.

14. Kim HD, Hesterman J, Call T, Magazu S, Keeley E, Armenta K, Kronman H, Neve RL, Nestler EJ, Ferguson D. SIRT1 Mediates Depression-Like Behaviors in the Nucleus Accumbens. J Neurosci 2016; 36(32): 8441 8452.

15. Heller EA, Hamilton PJ, Burek DD, Lombroso SI, Pena CJ, Neve RL, Nestler EJ. Targeted Epigenetic Remodeling of the Cdk5 Gene in Nucleus Accumbens Regulates Cocaine- and Stress-Evoked Behavior. J Neurosci 2016; 36(17): 4690-4697.

16. Plagg B, Ehrlich D, Kniewallner KM, Marksteiner J, Humpel C. Increased Acetylation of Histone $\mathrm{H} 4$ at Lysine 12 (H4K12) in Monocytes of Transgenic Alzheimer's Mice and in Human Patients. Curr Alzheimer Res 2015; 12(8): 752-760.

17. Liu D, Qiu HM, Fei HZ, Hu XY, Xia HJ, Wang LJ, Qin LJ, Jiang $X H$, Zhou $Q X$. Histone acetylation and expression of mono-aminergic transmitters synthetases involved in CUS-induced depressive rats. Exp Biol Med (Maywood) 2014; 239(3): 330-336.

18. Malberg JE, Blendy JA. Antidepressant action: to the nucleus and beyond. Trends Pharmacol Sci 2005; 26(12): 631-638.

19. de Sousa CN, Meneses LN, Vasconcelos GS, Silva MC, Da SJ, Macedo D, de Lucena DF, Vasconcelos SM. Reversal of corticosterone-induced BDNF alterations by the natural antioxidant alpha-lipoic acid alone and combined with desvenlafaxine: Emphasis on the neurotrophic hypothesis of depression. Psychiatry Res 2015; 230(2): 211-219.

20. Wang $H$, Zhao $Y$, Wang $Y J$, Song $L$, Wang JL, Huang $C$, Zhang $W$, Jiang $B$. Antidepressant-like effects of tetrahydroxystilbene glucoside in mice: Involvement of BDNF signaling cascade in the hippocampus. Cns Neurosci Ther 2017; 23(7): 627-636.

21. Liao L, Zhang $X D$, Li J, Zhang ZW, Yang CC, Rao CL, Zhou CJ, Zeng L, Zhao LB, Fang L, Yang DY, Xie P. Pioglitazone attenuates lipopolysaccharide-induced depression-like behaviors, modulates NF-kappaB/IL6/STAT3, CREB/BDNF pathways and central serotonergic neurotransmission in mice. Int Immunopharmacol 2017; 49: 178-186.

22. Jianhua F, Wei W, Xiaomei L, Shao-Hui W. Chronic social defeat stress leads to changes of behaviour and memory-associated proteins of young mice. Behav Brain Res 2017; 316: 136-144.

Trop J Pharm Res, September 2021; 20(9): 1880 